\title{
On the Space-Time Interpretation of Classical Canonical Systems II : Relativistic Canonical Systems
}

\author{
K. Drühl
}

Max-Planck-Institut zur Erforschung der Lebensbedingungen der wissenschaftlich-technischen Welt, D-8130 Starnberg, Federal Republic of Germany

\begin{abstract}
Relativistic canonical systems and their symmetries are defined and classified within the class of canonical systems treated in a previous paper. Their algebra of variables contains a subset of "monotone" variables which satisfy a certain uniqueness condition and are later shown to increase strictly in the course of the dynamical evolution of the system on all physically acceptable states. This leads to a unique space-time interpretation of relativistic canonical systems. Finally we study space-time factorizations of such systems and introduce the appropriate notion of states. For a certain simple class of states the theory is shown to describe the motion of relativistic matter in some external gravitational and electromagnetic field.
\end{abstract}

\section{Introduction}

In this paper we shall study a certain class of canonical systems, the general theory of which we have developed in [1]. Let us briefly state the basic notions and results obtained there.

A canonical system is an ordered set containing a canonical manifold $M$ (with canonical form $\Omega$, see [2]) and a canonical vectorfield $Y$ on $M$ (the kinematical vectorfield). The algebra $\mathfrak{A}(M)$ of differentiable functions on $M$ (these are called variables) contains a subalgebra $\mathfrak{A}_{0}$ which is required to satisfy a set of Kinematical Axioms: Under the Poisson bracket operation $\mathfrak{A}_{0}$ is maximal commutative and is mapped to itself by variables from the subset $Y\left(\mathfrak{H}_{0}\right)$. Both $\mathfrak{A}_{0}$ and $Y\left(\mathfrak{U}_{0}\right)$ have only the zero variable in common and determine the differentiable structure on $M$ (such sets of functions which define a differentiable structure on $M$ are called sufficient sets).

The Hamiltonian vectorfields generated by variables in $\mathfrak{A}_{0}$ define a quotient manifold $N$ of integral submanifolds in $M$. To any variable $A$ in $\mathfrak{A}_{0}$ there corresponds a unique differentiable function $A^{*}$ on $N$, and vice versa. The vectorfields $X$ on $N$ are in bijective correspondence with variables $P_{X}$ in some submodule of functions $\mathfrak{A}_{1}$ :

$$
\left\{P_{X}, A\right\}^{*}=X\left(A^{*}\right) ; A \text { in } \mathfrak{A}_{0}, P_{X} \text { in } \mathfrak{U}_{1} .
$$


There exists a unique diffeomorphism of $M$ with the cotangent bundle $L^{*}(N)$ over its quotient, and the canonical structure is completely determined by the equations:

i) $\{A, B\}=0$,

ii) $\left\{P_{X}, A\right\}^{*}=X\left(A^{*}\right)$,

iii) $\left\{P_{X}, P_{X_{1}}\right\}=P_{\left[X, X_{1}\right]}+F\left(X, X_{1}\right)$

for $A, B$ in $\mathfrak{A}_{0} ; P_{X}, P_{X_{1}}$ in $\mathfrak{U}_{1}$. The function $F\left(X, X_{1}\right)$ is in $\mathfrak{A}_{0}$ and defines a closed differential 2-form $\omega_{F}$ on $N$ by:

iv) $F\left(X, X_{1}\right)^{*}=\omega_{F}\left(X, X_{1}\right)$.

Finally one obtains a unique nowhere degenerate symmetric tensorfield $g$ on $N$ by:

i) $g\left(d A^{*}, d B^{*}\right)=\{Y(A), B\}^{*}$,

and the kinematical vectorfield $Y$ is given by:

ii) $Y(F)=\{T, F\}+Y^{0}(F), F$ in $\mathfrak{A}$,

where $2 T$ is the function on $L^{*}(N)$ corresponding to $g$, and $Y^{0}$ vanishes on $\mathfrak{A}_{0}$.

The subclass of canonical systems we study here is singled out by an additional set of Relativistic Axioms; these systems are called relativistic canonical systems. The most important of these axioms postulate the existence of "monotone" variables and a certain uniqueness condition for them.

In Chapter 2 relativistic canonical systems and their symmetries are defined and classified. By studying the trajectories defined by the kinematical vectorfield the notion of permissible integralcurves and of a physical submanifold are introduced. Monotone variables behave in a strictly monotone way along any trajectory in a physical submanifold. They should hence be considered as time-like variables.

Finally the most important Relativistic Axiom is deduced from the postulate that the initial value problem for the kinematical vectorfield shall admit a unique solution for any initial surface defined by some monotone variable in the physical submanifold. This leads to a unique space-time interpretation of relativistic canonical systems, according to which the quotient manifold $N$ must be considered as the space-time of general relativity.

In Chapter 3 we study space-time factorizations, which provides some motivation for our notion of states. These are defined as positive linear functionals on variables with compact support, which result from integration over hypersurfaces of constant time. The differential forms required for this are called state forms. They describe completely the motion of the matter present and define the current vector and energy-momentum tensor of the matter. Some aspects of $Y$-invariant forms are discussed. The detailed theory of state forms and the resulting equations of motion for the classical fields will be developed in a subsequent paper.

\section{Relativistic Canonical Systems and Their Symmetries}

In this chapter we shall study a certain class of canonical systems, for which a complete kinematical interpretation can be obtained. According to this interpretation the quotient manifold $N$ we have constructed in [1] will appear to be 
identical to the space-time of general relativity. These systems will be called relativistic canonical systems. They satisfy an additional set of Relativistic Axioms, concerning the dynamical vectorfield of the system. The concepts necessary to formulate these axioms are introduced below; they will be discussed thoroughly later on.

Consider the relation between functions in $\mathfrak{A}_{1}$ and vectorfields on the quotient $N$ established by (1.1). This relation may be extended to symmetric tensorfields of higher order. Denote by $\mathfrak{A}_{k}$ the $\mathfrak{A}_{0}$-module generated locally in $\mathfrak{A}$ by monomials of degree $k$ in functions from $Y\left(\mathfrak{U}_{0}\right)$. By $\mathfrak{A}^{r}$ we denote the corresponding module generated by all sets $\mathfrak{U}_{k}$ with $k \leqq r$. We state the following Proposition without proof; this would be analogous to the proof of Proposition 2.4 in [1].

Proposition 2.1. Let $F$ be in $\mathfrak{A}$, then $F$ is in $\mathfrak{A}^{k}$ if and only if:

$$
\xi_{d A_{1}} \circ \xi_{d A_{2}} \circ \ldots \circ \xi_{d A_{k}}(F) \text { is in } \mathfrak{U}_{0}
$$

for any set $\left(A_{1}, \ldots, A_{k}\right)$ of functions from $\mathfrak{U}_{0}$. For any $F$ in $\mathfrak{U}^{k}$ there exists a unique symmetrical tensorfield $D_{F}^{k}$ defined by:

$$
D_{F}^{k}\left(d A_{1}^{*}, \ldots, d A_{k}^{*}\right)=(-1)^{k} / k ! \xi_{d A_{1}}{ }^{\circ} \ldots \circ \xi_{d A_{r}}(F)^{*} .
$$

The kernel of the map $D^{k}$ in $\mathfrak{A}^{k}$ is the submodule $\mathfrak{A}^{k-1}$.

Let $T^{k}$ be any symmetric tensorfield of degree $k$. There exists a unique function $P_{T}^{k}$ in $\mathfrak{A}_{k}$ which is mapped to $T^{k}$ by the map $D^{k}$. Denoting the commutative tensormultiplication by " $\otimes$ " one has for the maps $D$ and $P$ :

$$
D_{F, G}^{i+k}=D_{F}^{i} \otimes D_{G}^{k}, P_{T \otimes S}^{i+k}=\dot{P}_{T}^{i} \cdot P_{S}^{k} .
$$

Identifying the isomorphic algebras $\mathfrak{A}_{0}$ and $\mathfrak{A}(N)$ the maps $D^{k}$, and $P_{k}$ are easily seen to be $\mathfrak{U}_{0}$-linear. Hence we obtain a unique isomorphism $D$ of the subalgebra $\mathfrak{U}^{\infty}$ of locally finite sums of functions from all sets $\mathfrak{U}_{k}$ with the symmetric tensorfield algebra over N.D is an isomorphism of commutative algebras over the ring of functions $\mathfrak{A}_{0}$.

With an obvious shorthand notation we have the following relations in $\mathfrak{I}^{\infty}$ :

i) $\left\{\mathfrak{A}^{k}, \mathfrak{A}^{j}\right\} \subset \mathfrak{A}^{k+j-1}$

ii) $Y\left(\mathfrak{U}_{0}\right) \subset \mathfrak{A}_{1}, Y\left(\mathfrak{Q}^{k}\right) \subset \mathfrak{A}^{k+1}$.

It follows that $Y$ is a derivation on the subalgebra $\mathfrak{A}_{0} \cup \mathfrak{H}^{\infty}$. The first relativistic axiom will require that $Y$ be a derivation on subalgebra $\mathfrak{A}^{\infty}$ alone, without its ring of coefficients $\mathfrak{A}_{0}$. This means that $Y$ should act monotonely with respect to the graduation in $\mathfrak{U}_{0} \cup \mathfrak{U}^{\infty}$.

The second axiom concerns the existence of "monotone" variables on $M$, and a certain uniqueness condition imposed on them. These variables will appear to increase strictly in the course of the dynamical evolution of the system for all physically acceptable states. They may therefore be considered as time-like variables.

Definition 2.1. Let $A$ be in $\mathfrak{A}_{0}$, and $U$ an open submanifold of the quotient $N$. $A$ is called monotone over $U$ if

i) $\{Y(A), A\}^{*}>0$ in $U$. 
The set of monotone variables over $U$ is denoted by $K(U)$.

$A$ is called monotone if it is monotone over $N$. Let $A$ be monotone over $U$. The positive set $K_{A}(U)$ associated to $A$ over $U$ is defined as the set of all monotone variables $B$ over $U$ for which:

ii) $\{Y(B), A\}^{*}>0$ in $U$.

Relativistic Axioms: Let $\left(L^{*}(N), \Omega, Y\right)$ be a canonical system.

R.i) $Y\left(\mathfrak{U}_{1}\right) \subset \mathfrak{U}_{1} \cup \mathfrak{U}_{2}$.

R.ii) There exists a monotone variable.

R.iii) For any open submanifold $U$ of $N$ and any variable $A$ monotone over $U$ one has: $K_{A}(U)=K_{B}(U)$ for any $B \in K_{A}(U)$.

A kinematical system satisfying these Axioms is called a relativistic canonical system (RCS).

Proposition 2.2. The axiom R.iii) is equivalent to:

R.iii') For any open submanifold $U$ of $N$ and $A, B$ in $K(U)$ either : $\{Y(A), B\}^{*}>0$, or: $\{Y(A), B\}^{*}<0$ in $U$.

Proof. Denote by $g$ the nowhere degenerate tensorfield defined in $(1.3, \mathrm{i})$. Assume that for some $q$ in $U: g\left(d A^{*}, d B^{*}\right)(q)=0$. Choose an open submanifold $V$ at $q$ in $U$ such that for some constant $d$ one has:

$$
g\left(d A^{*}, d A^{*}\right), g\left(d B^{*}, d B^{*}\right) \geqq d ; g\left(d A^{*}, d B^{*}\right)<d .
$$

Then we have for the function $C=A+B: C \in K_{A}(V), C \in K_{B}(V)$, however $A$ is not in $K_{B}(V)$ contradicting R.iii). Conversely assume this situation is met for three monotone variables $A, B, C$ over $V$. Then for some $q$ in $V$ we have:

$$
g\left(d A^{*}, d C^{*}\right)(q)=d>0 ; g\left(d A^{*}, d B^{*}\right)(q)=-b<0 .
$$

However the function $D=d \cdot B+b \cdot C$ is in $K(V)$, and we have $g\left(d A^{*}, d D^{*}\right)(q)=0$, contradicting R.iii'). QED.

Theorem 2.1. Let $\left(L^{*}(N), \Omega, Y\right)$ be a relativistic canonical system. The nowhere degenerate tensorfield $g$ on $N$ defined by $Y$ is of signature $(1, n-1)$ everywhere. The dynamical vectorfield $Y$ is Hamiltonian: $Y=\xi_{d H}$, where $2 H$ is the function on $L^{*}(N)$ defined by $g: 2 H=P_{g}^{2}$. In any associated chart $\left(U ; Q^{i}, P_{r}\right)$ we have

$$
H=\frac{1}{2} \sum_{i, r} G^{i r} P_{i} P_{r} ; G^{i r}=\left\{Y\left(Q^{i}\right), Q^{r}\right\} .
$$

Proof. By R.ii) there is a monotone variable $A$, hence $g\left(d A^{*}, d A^{*}\right)>0$ on $N$. By Proposition 2.2 there cannot exist a differential $d B_{q}^{*}$, such that $g\left(d B_{q}^{*}, d B_{q}^{*}\right)>0$ and $g\left(d A_{q}^{*}, d B_{q}^{*}\right)=0$. Hence $g$ is of signature $(1, n-1)$. By [1], Theorem 3.2 we have to show that $Y^{0}$ vanishes. However $\left\{Y^{0}\left(P_{X}\right), B\right\}=Y^{0}\left(\left\{P_{X}, B\right\}\right)-\left\{P_{X}, Y^{0}(B)\right\}=0$ for $B$ in $\mathfrak{A}_{0}$, hence $Y^{0}\left(P_{X}\right)$ is in $\mathfrak{A}_{0}$. On the other hand $\xi_{d T}\left(P_{X}\right)$ is in $\mathfrak{A}_{1} \cup \mathfrak{A}_{2}$, and the same is true for $Y\left(P_{X}\right)$ by R.i). Hence $Y^{0}\left(P_{X}\right)=0$, and thus $Y^{0}=0$. QED.

We obtain a physical interpretation for any given RCS if we interpret the integralcurves of $Y$ as defining the trajectories of physical particles. Their equations ([1], (3.4)) then describe the motion of these particles of unit charge in the gravitational field described by the tensorfield $g$ and the external electromagnetic field described by the closed 2 -form $\omega_{F}$. Note that no additional dynamicle principle 
is required for this result apart from the Kinematical and Relativistic Axioms. We shall now consider this "external field interpretation" somewhat more closely. The important Relativistic Axiom R.iii) will be shown to result from a physical postulate concerning the initial value problem for the integral curves of $Y$.

Let $\gamma$ be some integral curve of $Y$ with $p$ on $\gamma$. By projecting onto the quotient $N$ we obtain a corresponding curve $\gamma^{*}$ in $N$ which we shall call a trajectory through $q=\pi(p)$. The tangentvector defined by this trajectory at $q$ is just the projection of $Y_{p}$ :

$$
Y_{p}^{*}\left(A^{*}\right)=Y_{p}(A)=Y(A)\left(q, \omega_{q}\right) .
$$

As $p$ varies over the fixed submanifold $p^{*}=\pi^{-1}(q)$ in $L^{*}(N)$, the corresponding projections vary over the set of directions into which trajectories through $q$ may go. By (2.2) these directions define the corresponding values for all functions in $Y\left(\mathfrak{H}_{0}\right)$ on $p^{*}$.

Since these values uniquely determine the points on $p^{*}$, and hence the integralcurves through $p^{*}$, we conclude that any trajectory is uniquely determined by a single point on it plus its direction at that point.

Now consider the values which variables like $P_{X}$ take along some fixed integral curve $\gamma$. It is not difficult to see that except at points where $P_{X}=0$ for all $X$ there exists a function $B$ in $\mathfrak{A}_{0}$ such that:

$$
P_{X}=X\left(B^{*}\right) \text { along } \gamma \text { resp. } \gamma^{*} \text {, }
$$

and $B^{*}$ is determined uniquely up to a constant along the corresponding part of $\gamma^{*}$. We shall call $B$ a parameter associated to $\gamma$, and the points on $\gamma$ are just given by $\left(q, d B_{q}^{*}\right), q$ on $\gamma^{*}$. However from (2.2) we obtain:

$$
Y_{p}^{*}\left(A^{*}\right)=Y(A)\left(q, d B_{q}^{*}\right)=\{Y(A), B\}(p)=\{Y(B), A\}(p) .
$$

Thus the integral curve through $q$ of the vectorfield generated by the function $Y(B)$ is just the trajectory through $q$. In this sense we may say that the trajectories on $N$ are generated by the first order derivatives of their associated parameters. This connection between the dynamical evolution of the system and the transformations generated by the momenta variables $P_{X}$ is the essential content of the kinematical axiom K.iv) stated in [1].

We shall now for any integral curve consider the change of its associated parameter along the corresponding trajectory. This will lead to the notion of "permissible integral curves".

Definition 2.2. Any integral curve $\gamma$ of $Y$ is called permissible if any associated parameter increases along the corresponding trajectory:

i) $Y_{p}^{*}\left(B^{*}\right)=\{Y(B), B\}^{*}(q)>0$.

Any maximal open submanifold of $L^{*}(N)$ containing only permissible curves is called a physical manifold $L_{0}^{*}(N)$.

We realize that the parameters associated to permissible curves are exactly the variables which are monotone over some open submanifold $U$. For any canonical system an integral curve is permissible if and only if the function defined by the tensorfield $g$ is non-negative along this curve (see Theorem 2.2 of [1]). In 
particular non-relativistic particle mechanics may be characterized by the fact that all curves are permissible. We shall now specialize again to RCS and derive the Relativistic Axiom R.iii) from a simple postulate. This postulate requires that the initial value problem for the vectorfield $Y$ has a solution in any physical manifold for all submanifolds defined by monotone variables.

Initial Value Problem: Let $U$ be an open submanifold of $L^{*}(N)$, and $U_{f}$ a submanifold in $U$ defined by an equation $F=f=$ const, $F$ in $\mathfrak{A}(U)$. Does there exist an open submanifold $V$ in $U$ containing $U_{f}$ such that the following equivalent statements hold:

i) Through any point in $U_{f}$ there passes a unique integral curve of $Y$ in $V$ which is not tangential to $U_{f}$, and different points correspond to different curves in $V$.

ii) For any function $G_{f}$ in $\mathfrak{A}\left(U_{f}\right)$ there exists a unique $Y$-invariant function $G$ in $\mathfrak{A}(V)$ such that:

$$
Y(G)=0 \text { in } V ; G=G_{f} \text { on } U_{f} .
$$

By considering a suitable chart at some point on $U_{f}([4] ; \mathrm{I}, \S 3.1)$ we realize that the integral curves of $Y$ are just the characteristic curves of the partial differential equation resulting from ii) in that chart. The necessary and sufficient condition for the Initial Value Problem of that differential equation posed by the submanifold $U_{f}$ to have a solution (see for example [4], 2. Kap., §2) may be reformulated as:

$$
Y(F) \neq 0 \text { on } U_{f} .
$$

By covering $U_{f}$ with charts as above it is not difficult to demonstrate this result for all of $U_{f}$. Hence the Initial Value Problem has a solution if and only if $Y(F) \neq 0$ on $U_{f}$, and thus either $Y(F)>0$ or $Y(F)<0$, since $U_{f}$ is connected.

Postulate 2.1. Let $U$ be an open submanifold of $N$, and $L_{0}^{*}(U)$ any physical submanifold of $L^{*}(U)$. Then for any submanifold in $L_{0}^{*}(U)$ defined by an equation $B=b=$ const with monotone variable $B$ over $U$ the Initial Value Problem has a solution.

Proposition 2.3. Any kinematical system satisfying the Initial Value Postulate 2.1 satisfies the Relativistic Axiom R.iii).

Proof. For any $A, B$ monotone over $U$ the point $\left(q, d A_{q}^{*}\right)$ with $q$ in $U$ is in some $L_{0}^{*}(U)$, hence the Initial Value Problem posed by $B$ at $\left(q, d A_{q}^{*}\right)$ has a solution, and:

$$
Y(B)\left(q, d A_{q}^{*}\right)=\{Y(B), A\}^{*}(q)>0,
$$

or

$$
\{Y(B), A\}^{*}(q)<0 .
$$

Since $U$ is connected, one of these inequalities must hold on all of $U$, and by Proposition 2.2 the Axiom R.iii) is satisfied. QED.

For a relativistic canonical system we may define two physical manifolds.

Proposition 2.4. Let $\left(L^{*}(N), \Omega, Y\right)$ be a relativistic canonical system with Hamiltonian $H$ and monotone variable $A$. Then any physical manifold is equal to one of the 
following disjoint open submanifolds.

i) $L_{>}^{*}=(p \mid H(p)>0, Y(A)>0)$.

ii) $L_{<}^{*}=(p \mid H(p)>0, Y(A)<0)$.

The proof is immediate from Proposition 2.2. We may conclude from this the important result that the Initial Value Problem posed by $A$ has a solution in any physical manifold. Choosing a definite physical manifold, say $L_{>}^{*}$ amounts to choosing a definite orientation of time with respect to the integration parameter of $Y$, and turns $A$ into a positive timelike variable. Furthermore it is not difficult to see that for any $B$ monotone over some $U$ we have:

$$
Y(B)>0 \quad \text { or } \quad Y(B)<0 \quad \text { in } \quad L_{0}^{*}(U)
$$

hence the Initial Value Problem posed by $B$ has a solution in that part of $L_{0}^{*}$ projecting onto $U$.

We shall finally discuss the notion of symmetry for relativistic canonical systems. Let $\varphi$ be a canonical transformation on $L^{*}(N)$ leaving $Y$ invariant:

$$
Y^{\phi}=\phi \circ Y \circ \phi^{-1}=Y ; \phi(F)=F \circ \varphi \quad \text { for } \quad F \in \mathfrak{U} .
$$

It is not difficult to check that for any such transformation the subalgebra $\phi\left(\mathfrak{U}_{0}\right)$ satisfies all Kinematical Axioms if $\mathfrak{\mathfrak { A }}_{0}$ does. However $\phi\left(\mathfrak{U}_{0}\right)$ will in general be different from $\mathfrak{U}_{0}$. We call $\phi$ a symmetry of the relativistic canonical system considered if in addition to (2.2) it permutes $\mathfrak{U}_{0}$ :

$$
\phi: \mathfrak{U}_{0} \rightarrow \mathfrak{H}_{0} .
$$

It follows from (2.3) that $\varphi$ permutes the integral submanifolds generated by $\mathfrak{A}_{0}$, and hence induces a diffeomorphism $\varphi^{0}$ on the quotient $N$.

Theorem 2.2. Let $\phi$ be a symmetry of the relativistic kinematical system $\left(L^{*}(N), \Omega, Y\right)$. Then

i) $\phi^{0}[g]=g$,

ii) $\phi^{0}\left[\omega_{F}\right]=\omega_{F}$,

where $\omega_{F}$ and $g$ are defined as in (1.2) and (1.3), and $\phi^{0}$ corresponds to the induced diffeomorphism $\varphi^{0}$ on $N$. The symbols in i) and ii) denote the corresponding transformed quantities.

Conversely let $\varphi^{0}$ be a diffeomorphism on $N$ satisfying i) and ii). Then there exists a unique symmetry $\phi$ inducing $\varphi^{0}$.

Proof. We have for any $A, B$ in $\mathfrak{U}_{0}$

$$
\begin{aligned}
\phi^{0}\left(g\left(d A^{*}, d B^{*}\right)\right) & =\phi(\{Y(A), B\})^{*} \\
& =\{Y(\phi(A)), \phi(B)\}^{*} \\
& =g\left(d\left(\phi^{0}\left(A^{*}\right)\right), d\left(\phi^{0}\left(B^{*}\right)\right)\right.
\end{aligned}
$$

which is equivalent to i). Furthermore we obtain from this

iii) $\phi\left(P_{X}\right)=P_{\phi^{0}[X]}$ 
for any $P_{X}$ in $Y\left(\mathfrak{U}_{0}\right)$, and hence for any $P_{X}$ in $\mathfrak{U}_{1}$. By a similar calculation as above we conclude for the function $F\left(X, X_{1}\right)$ defined in $(1.2$, iii):

iv) $\phi\left(F\left(X, X_{1}\right)=F\left(\phi^{0}[X], \phi^{0}\left[X_{1}\right]\right)\right.$,

which is equivalent to ii). Now let $\varphi^{0}$ be a diffeomorphism satisfying i) and ii). Consider the map $\phi: \mathfrak{U} \rightarrow \mathfrak{U}$ defined by:

$$
\begin{array}{lll}
\phi(A)^{*}=\varphi^{0}\left(A^{*}\right), & A & \text { in } \mathfrak{U}_{0} ; \\
\phi\left(P_{X}\right)=P_{\phi^{0}[X]}, & P_{X} & \text { in } \mathfrak{U}_{1} .
\end{array}
$$

It is not difficult to check that $\phi$ is well defined and corresponds to a permutation of the points in $L^{*}(N)$, hence defines a diffeomorphism $\varphi$ on $M$. By (1.2) and iv) we may conclude that $\phi\{F, G\}=\{\phi(F), \phi(G)\}$ for $F, G$ in the sufficient set $\mathfrak{U}_{0} \cup \mathfrak{A}_{1}$, hence $\varphi$ is canonical. Finally we get:

$$
\varphi(Y(A)=Y(\phi(A)),
$$

and by calculating the image under $\phi$ of the expression for $Y(\{Y(A), B\}) ; A, B$ in $\mathfrak{A}_{0}$; we obtain:

v) $\phi\left(Y^{2}(A)\right)-Y^{2}(\phi(A))$ is in $\mathfrak{A}_{0}$.

However $\phi$ maps every homogeneous subspace $\mathfrak{U}_{k}$ to itself, hence by R.i) the term v) vanishes and we have:

$$
\phi(Y(F))=Y(\phi(F))
$$

for $F$ in $\mathfrak{A}_{0} \cup Y\left(\mathfrak{U}_{0}\right)$, which implies that $\phi[Y]=Y$. QED.

\section{Space-Time Decomposition and States}

In this chapter we shall define the notion of states for relativistic canonical systems, and discuss a certain simple classe of states. The detailed theory of states which comprises the equations of motion for the classical fields will be developed in a subsequent paper. It will be instructive to briefly study a certain decomposition of the classical algebra of variables beforehand, which corresponds to the distinction between timelike and spacelike variables.

Let $A$ be a monotone variable (R.ii), and $X$ be the associated vectorfield defined by:

$$
P_{X}=\{Y(A), A\}^{-1} Y(A) ;\left\{P_{X}, A\right\}=1 .
$$

The canonical vectorfields defined by $P_{X}$ and $A$ commute, and we shall assume for simplicity that both generate one parameter groups. By arguing along similar lines as in [1] we may show that the set of maximal integral manifolds of both fields can be turned into a manifold $L_{R}^{*}$, which we call the reduced manifold. The algebra of differentiable functions on $L_{R}^{*}$ is isomorphic to the subalgebra:

$$
\mathfrak{A}_{R}=\left(F \quad \text { in } \quad \mathfrak{A} ;\left\{P_{X}, F\right\}=\{A, F\}=0\right) .
$$

The Poisson bracket maps $\mathfrak{A}_{R}$ to itself and is easily seen to induce a unique Poisson bracket on the reduced manifold. 
Any given integral manifold $w$ is diffeomorphic to $R^{2}$, with $A$ and $P_{X}$ defining global coordinates. On the physical submanifold $w_{0}=w \cap L_{0}^{*}$ we have:

i) $\partial H / \partial P=-\{A, H\}=Y(A)>0, P=P_{X}$;

hence we may introduce $H$ and $A$ as coordinates on $w_{0}$. Any variable $F$ in $\mathfrak{U}$ may thus on $L_{0}^{*}$ be considered as a unique function $F_{R}$ on $L_{R}^{*}$ depending on the additional parameters $H$ and $A$ :

ii) $F(p)=F_{R}(w ; H, A)$.

The vectorfields corresponding to differentiation with respect to these parameters are given by:

iii) $X_{H}=\partial / \partial H=-Y(A)^{-1} \xi_{d A}$

iv) $X_{A}=\partial / \partial A=\xi_{d P}-Y(A)^{-1} Y(P) \xi_{d A}$.

These vectorfields define a unique decomposition for any differential 1-form. By considering the form $d P$ we obtain:

i) $\omega=\omega_{R}+\omega\left(X_{H}\right) d H+\omega\left(X_{A}\right) d A$

ii) $d H=Y(A)\left(d P-(d P)_{R}\right)-Y(P) d A$

iii) $Y(F)=\Lambda(d H, d F)=\Lambda\left(d H,(d F)_{R}+X_{A}(F) d A\right)$

$$
\begin{aligned}
& =Y(A) \cdot \Lambda\left(d P-(d P)_{R},(d F)_{R}+X_{A}(F) d A\right) \\
& =Y(A)\left[-\Lambda\left((d P)_{R},(d F)_{R}\right)+X_{A}(F)\right] .
\end{aligned}
$$

Now the differential form $(d F)_{R}$ is just the differential of the function $F_{R}$, considered as a function on $L_{R}^{*}$ alone. Hence we obtain the following decomposition.

iv) $Y(A)^{-1} Y=-\left\{P_{R}, \ldots\right\}_{R}+\partial / \partial A$.

The first term is tangential to any manifold $w_{0}$, and hence defines a vectorfield on $L_{R}^{*}$ which depends upon the additional parameters $H$ (=mass) and $A$ (=time).

This field is just the canonical field generated by the Hamiltonian $P_{R}$ with respect to the induced Poisson bracket on $L_{R}^{*}$. We emphasize that $P_{R}$ must be considered here as a function on $L_{R}^{*}$ only, the parameter $H$ and $A$ being kept fixed. The second term is just the partial derivative with respect to the time $A$. In particular we obtain for any $F$ invariant under $Y$ :

$\partial F_{R} / \partial A=\left\{P_{R}, F_{R}\right\}_{R}$.

This relation has led to the wide spread belief that the momentum associated to a timelike variable is the Hamiltonian with respect to that variable. However this is not the case if we consider the momentum $P$ as a function of all relevant parameters as it is necessary for a fully covariant formulation. The actual Hamiltonian is the mass-squared-variable $H$. The integration parameter associated to $H$ is easily seen to be (mass) $)^{-1} \times$ proper time); it is related in a strictly associated way on the physical manifold to any timelike variable. Finally we emphasize that the reduced manifold we have constructed is a quotient manifold rather 
than a constraint submanifold [3]. This allows us to treat the mass as a genuine additional variable, rather than as some fixed constant. The constraint surface corresponding to some fixed value $h$ of $H$ is given by the equation:

$$
P_{X}=\left(P_{X}\right)_{R} \quad \text { for } A \text { arbitrary, } H=h=\text { const. }
$$

Let us now define the notion of states appropriate for our theory. In the framework of the reduced manifold states would correspond to measures on $L_{R}^{*}$, depending upon the additional parameters $A$ and $H$. If we allow in addition for integration over the mass variable $H$, the analogue in the original manifold would be a set of surface measures defined on submanifolds of constant time.

Proposition 3.1. Let $A$ be a monotone over $U$. Any submanifold $V_{a}$ defined by an equation $A=a=$ const in $\pi^{-1}(U)_{0}$ is orientable.

Since $Y(A)>0$ on $\pi^{-1}(U)_{0}=\pi^{-1}(U) \cap L_{0}^{*}$ the $(2 n-1)$-form $\mu(Y, \ldots)$ defines an orientation on $V_{a}$, where $\mu$ is the canonical $2 n$-form on $L_{0}^{*}$ (see [2]). We shall always integrate $(2 n-1)$-forms with respect to this orientation over submanifolds like $V_{a}$ which are defined by monotone variables.

Definition 5.1. A state form is a closed $(2 n-1)$-form $\omega$ on $L_{0}^{*}$ such that:

$$
\int_{V_{a}} F \cdot \omega \geqq 0
$$

for any positive $F$ with compact support and for any integral taken over some submanifold defined by some monotone variable $A$ as in Proposition 3.1.

Consider the dual of the state form $\omega$, which is the vectorfield $\omega^{*}$ defined by:

i) $\mu\left(\omega^{*}, \ldots\right)=\omega(\ldots)$.

It is not difficult to check that $\omega$ being a state form is equivalent to:

ii) $\omega^{*}(A) \geqq 0$ in $\pi^{-1}(U)_{0}$ for $A$ monotone over $U$.

If $\omega$ is some state form then a given multiple $F \cdot \omega$ with $F \geqq 0$ is a state form if and only if

iii) $\omega^{*}(F)=0$,

and hence if $F$ is constant along the integral curves of $\omega^{*}$. These curves may be interpreted to define the trajectories of massive particles. The discussion of such trajectories in Chapter 2 corresponds to the special case where $\omega^{*}$ is proportional to $Y$; we call such forms $Y$-invariant.

Definition 3.2. Let $\omega$ be a state form and $A$ be monotone. The state defined by $\omega$ at time $A=a$ is given by the positive linear map:

$$
S(\omega, a): G \rightarrow \int_{A=a} G \cdot \omega
$$

for variables with compact support, where the integral is taken over the submanifold defined by $A=a$ in $L_{0}^{*}$. 
We may express any state by integration in the reduced manifold:

$$
\int_{A=a} G \cdot \omega=\int_{0}^{\infty} d H \int_{L_{R}^{*}}\left[G \cdot \omega^{*}(A) \cdot Y(A)^{-1}\right]_{R}(H, a) \mu_{R}(H, a)
$$

where $\mu_{R}$ is the canonical volume element in the reduced manifold: $\mu=d A \wedge d P \wedge$ $\omega_{R}(H, A)$. The function $\omega^{*}(A) \cdot Y(A)^{-1}$ is the corresponding density. For $Y$ invariant state forms it is just the function $F$ defined by $\omega^{*}=F \cdot Y$ with $Y(F)=0$ [see Eq. (3.5)].

In order to write down equations for the classical fields we need quantities like the current and the energy-momentum tensor, which serve as sources for the classical fields. These quantities may be obtained for any given state form $\omega$ by first integrating at any point $q$ in $N$ over that part $K_{q}$ of the cotangent space contained in the physical manifold $L_{0}^{*}$ :

Proposition 3.2. Let $\omega$ be a $(2 n-1)$-form on $L^{*}$. Then:

i) $\omega_{0}(X)(q)=\int_{K_{q}} \omega\left(\xi_{X}, \ldots\right)$

defines a $(n-1)$-form on $N$, where $\xi_{X}$ is the canonical vectorfield generated by $P_{X}$. (We have used a single symbol $X$ to denote a set of $n-1$ vectorfields on $N$.) If $\omega$ is closed and vanishes on the boundary of $K_{q}$ the form $\omega_{0}$ is closed. For any submanifold $V_{a}$ as in Proposition 3.1 one has:

ii) $\int_{V_{a}} \omega=\int_{\pi\left(V_{a}\right)} \omega_{0}$.

If we assume that $N$ is orientable, we may define dual objects for differential forms on $N$ by means of the metric $g$. The dual of the form $\omega_{0}$ in Proposition 3.2 is a vectorfield $J(\omega)$ which we call the current defined by $\omega$. Within some given chart at a point in $\pi\left(V_{a}\right)$ we may write the integral ii) in terms of the components of $J(\omega)$ and of the surface differential form $d \sigma$ :

i) $\int_{V_{a}} \omega=\int_{\pi\left(V_{a}\right)} \sum_{k} d \sigma_{k} J^{k}(\omega)$.

Now consider forms like $\omega^{\prime}=G \cdot \omega$, where $\omega$ is some state form. We have:

ii) $J(A \cdot \omega)=A^{*} J(\omega)$ for $A$ in $\mathfrak{A}_{0}$

iii) $J\left(P_{X} \cdot \omega\right)=T(X ; \omega)$ for $P_{X}$ in $\mathfrak{U}_{1}$.

The current $J(\omega)$ is conserved since $\omega$ is closed. The map $T: X \rightarrow T(X ; \omega)$ is easily seen to be an $\mathfrak{A}(N)$-linear map of vectorfields on $N$, and hence to define a mixed vector-covector field of degree 2 . We call this the energy momentum field defined by $\omega$. Higher order tensorfields result from considering functions $G$ in $\mathfrak{A}_{k}$ $k>1$. For $Y$-invariant state forms we obtain particularly simple expressions for the components of these fields in some chart $\left(U, A^{k}\right)$ with associated chart $\left(\pi^{-1}(U)\right.$, $\left.A^{k}, P_{i}\right)$ on $L_{0}^{*}$ :

$\begin{aligned} \text { i) } J^{i}(\omega) & =\sum_{k} g^{i k} \sqrt{-d}^{-1} \int_{K_{q}} P_{k} \cdot F d \sigma(p) \\ \text { ii) } T_{j}^{i}(\omega) & =\sum_{k} g^{i k} \sqrt{-d}^{-1} \int_{K_{q}} P_{k} \cdot P_{j} \cdot F d \sigma(p)\end{aligned}$ 
where $d \sigma(p)$ is the volume element defined by the metric $\left(g^{i k}\right)$ in $K_{q}, F$ is the density function: $\omega=F \mu(Y, \ldots)$, and $d$ is the determinant of $\left(g^{i k}\right) ; n=4$. The tensor $T$ satisfies in this case the known conservation law for charged particles of fixed unit charge moving in the classical fields $g$ and $F$ :

iii) $\sum_{k} T_{i ; k}^{k}=\sum_{k} F_{i k} \cdot J^{k}$.

Let us summarize the important features of the concept of states we have introduced so far. In order to define a state two ingredients are required. The first is a state form $\omega$. This form completely describes the dynamics of the physical system as far as the motion of the matter is concerned. Furthermore by Proposition 3.2 and (3.9) it determines the current vector and energy-momentum tensor of the matter. The second ingredient is some space-like hypersurface in $N$. This can be chosen at will, corresponding to the non-uniqueness of the notion of equal time for different space-time events. Given both, expectation values may be formed by integrating the form over the corresponding hypersurface in $L_{0}^{*}$. Thus we may say that in order to represent observations in our theory we must specify both the variable to be observed and the instant of time with respect to some time variable at which the observation is to be made. Both aspects are necessary for a theory of observations in general relativity. To our opinion they are represented in a very natural and satisfactory way within our canonical framework.

In the present paper we have treated the dynamical vectorfield $Y$ and the canonical form $\Omega$ as fixed and independent of any particular state form. The equations of motion for the classical fields represented by these quantities will be treated in a subsequent paper, where we shall present a canonical theory of state forms.

This theory will allow for a discussion of charges and spin degrees of freedom as well.

\section{References}

1. Drühl,K.: On the space-time interpretation of classical canonical systems. I.: The general theory. Comm. math. Phys. 49, 277-288 (1976)

2. Jost, R.: Poisson brackets (An unpedagogical lecture), Rev. Mod. Phys. 36, 572-579 (1964)

3. Sniatycki,J., Tulczyjew, W. M.: Canonical dynamics of relativistic charged particles. Ann. Inst. H. Poincaré A, 15, 177-187 (1971)

Kijowski, J.: A finite-dimensional canonical formulation in the classical field theory. Commun. math. Phys. 30, 99-128 (1973)

4. Courant, R., Hilbert, D.: Methoden der Mathematischen Physik II. 2. Aufl. Berlin, Heidelberg, New York: Springer 1968

Communicated by J. Ehlers

Received June 2, 1975 